\title{
EDITORIAL
}

For reprint orders, please contact: reprints@futuremedicine.com

\section{Focusing on the fornix: can fornix diffusion tensor imaging measures be used to predict Alzheimer's disease?}

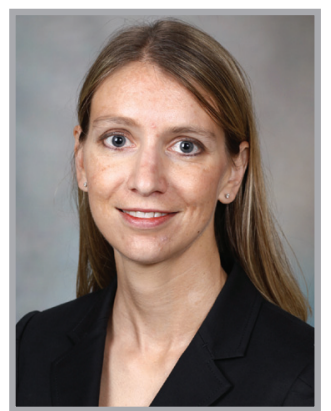

Michelle M Mielke* “...recent research suggests fornix white matter integrity may be a particularly sensitive neuroimaging biomarker of early Alzheimer's disease-related brain changes."
White matter alterations, including axonal and dendritic degeneration, demyelination and oligodendroglial cell death, are known pathological characteristics of the Alzheimer's disease (AD) brain at autopsy $[1,2]$. In vivo, white matter abnormalities have also been found with conventional MRI. However, this methodology is limited by the inability to delineate white matter tract organization, such that changes in specific white matter fiber bundles cannot be detected. More recently, the detection of subtle changes in white matter microstructure has become possible with the evolution of magnetic resonance diffusion tensor imaging (DTI). DTI quantifies the magnitude and directionality of water diffusion down the white matter tracts, and can reveal subtle abnormalities in white matter microstructure and brain connectivity. Fractional anisotropy (FA) and mean diffusivity (MD) are two DTI measures used to quantify white matter microstructure, by measuring the relative random motion of water in cerebral tissue. FA is a measure of anisotropic water diffusion, and reflects the degree of directionality of cellular structures within the fiber tracts, and therefore the structural integrity. MD, a measure of randomized mean water diffusion, represents a loss of barriers restricting the water motion, partial breakdown of tissue cytoarchitecture or demyelinating processes. Thus, higher values of FA and lower values of MD are thought to represent normal tissue cytoarchitecture.

Based on the characteristic pathological changes in postmortem AD brains [3], many DTI studies of AD patients have focused on the temporal lobes, cingulum, splenium and fornix. Of these, recent research suggests fornix white matter integrity may be a particularly sensitive neuroimaging biomarker of early ADrelated brain changes. The fornix is the predominant outflow tract of the hippocampus, and is critical for episodic memory functioning and anterograde memory. It is a central component of limbic projections and pathways that connect to the frontal lobes, and is preferentially affected early in the course of AD [3,4]. Notably, while a predominant outflow tract, the fornix also serves as a cholinergic input to the hippocampus and, therefore, could also be an important biomarker of $\mathrm{AD}$ progression.

*Division of Epidemiology, Department of Health Sciences Research, College of Medicine, Mayo Clinic, 200 1st Street SW, Rochester, MN 55905, USA; Tel.: + 1507284 5545; Fax: +1 507284 1516; mielke.michelle@mayo.edu

“...while a predominant outflow tract, the fornix also serves as a cholinergic input to the

hippocampus and, therefore, could also be an important biomarker of Alzheimer's disease progression." 
“...voxel-based

approaches often require multiple time-intensive preprocessing steps to ensure accurate image normalization and, therefore, are also unlikely to be clinically available."
The hypothesis that fornix changes occur early in the AD process is supported by a novel study of presymptomatic presenilin mutation carriers [5]. Compared with noncarriers, the presymptomatic carriers had significantly lower fornix FA, strongly suggesting that fornix white matter changes occur prior to memory loss and may be an early indicator of AD. Notably, in this study, FA of the cingulate bundle (comprising of the input tract to the hippocampus) did not significantly differ between mutation carriers and noncarriers, suggesting early specificity of changes in the fornix.

While the generalizability of these findings to sporadic $\mathrm{AD}$ may be questioned, additional research in nonmutation carriers also suggests fornix white matter is affected early in the course of $\mathrm{AD}$ and is therefore a predictor of disease progression. Among cognitively normal individuals, low fornix FA has been found to predict incident amnestic mild cognitive impairment (MCI) [6]. Decreasing fornix FA has also been associated with increasing AD severity [7,8], and can be used to predict those individuals who will progress from amnestic MCI to AD over a follow-up of 3 years [9]. Finally, fornix white matter integrity may be a useful diagnostic marker to differentiate AD. Recently, Zhuang et al. [10] reported that amnestic MCI patients had lower fornix FA and higher radial and axial diffusivity than either cognitively normal individuals or patients with nonamnestic MCI. Other studies have also reported reduced fornix $\mathrm{FA}$ in $\mathrm{AD}$ cases compared with patients with Lewy body dementia [11,12].

In parallel to this research examining fornix white matter as an AD biomarker, there has been a recent focus on the fornix for the treatment of AD. A recent Phase I trial of deep brain stimulation of the fornix in six AD patients reported possible improvements or slowing in the rate of cognitive decline with continuous stimulation over 1 year [13]. There were also sustained increases in both cortical-subcortical and cortical-hippocampal glucose metabolism over the stimulation period [14]. A larger Phase II study is underway. While there is debate as to whether the cognitive and lifestyle benefits will outweigh the cost and invasiveness of the procedures, the study does give further credence to the use of fornix white matter integrity as a biomarker for clinical progression in AD.

Together, the above-described findings suggest that fornix white matter integrity, as assessed with DTI, could be a sensitive clinical marker for the preclinical or early clinical diagnosis of AD and its rate of progression. So what are the limitations and where do we go from here? First, scanners with DTI capabilities are becoming widely available; however, additional methodological work is needed before fornix integrity can be implemented into the routine clinical diagnosis of AD patients. Currently, the image quantification procedure, which requires time-consuming and precise region-of-interest placement, is not practical for daily clinical routine. Automated voxel-based analyses have undergone reliability testing across nine different scanners and settings [15]. However, voxelbased approaches often require multiple timeintensive preprocessing steps to ensure accurate image normalization and, therefore, are also unlikely to be clinically available. An alternative, and promising, approach is the use of a visual assessment of reduced fornix FA, termed the 'fornix sign' [16]. In this method, the fornix is color coded from blue (low FA) to red (high FA). The 'fornix sign' was defined as the lack of any area with FA $<0.5$ indicating the yellow-red range and had 95\% inter-rater agreement [16]. Specificity for differentiating cognitively normal individuals from $\mathrm{AD}$ patients and predicting conversion from normal to MCI was $100 \%$. However, sensitivity was lower, including 56\% for differentiating cognitively normal individuals from $\mathrm{AD}$ patients and $67 \%$ for predicting which cognitively normal individuals would progress to MCI up to 3 years after follow-up. Regardless, the ability to predict conversion from cognitively normal to $\mathrm{MCI}$, and from MCI to AD, was notable, and suggests that the presence of a fornix sign could evolve into a clinically useful imaging biomarker of AD.

Second, while the above-described research suggests the fornix is a robust, early biomarker of $\mathrm{AD}$, some studies have reported that the changes in the cingulate bundle occur prior to changes in the fornix $[17,18]$. The posterior cingulum/precuneus sustains information transfer between the parahippocampal gyrus and the prefrontal cortex. Damage to this tract would lead to dysfunction of the network responsible for sustaining memory functions. While fornix FA better differentiated presenilin asymptomatic mutation carriers from noncarriers [5], as described above, there were some differences, albeit not significant, between the two groups in the cingulum. Among sporadic AD cases, it 
is likely that a combination of information from both regions will best predict incident $\mathrm{MCI}$ and progression to AD. If a 'posterior cingulate sign' could also be developed, it would be interesting to see whether the addition of this marker to the 'fornix sign' could increase the sensitivity for differentiating clinical groups and predicting progression. The visual assessment of both areas may be the most clinically feasible predictor for the clinical diagnosis of AD.
Financial \& competing interests disclosure

This work was supported by a grant from the National Institute on Aging (U01 AG006786) and the Walter S and Lucienne Driskill Foundation. The author has no other relevant affiliations or financial involvement with any organization or entity with a financial interest in or financial conflict with the subject matter or materials discussed in the manuscript apart from those disclosed.

No writing assistance was utilized in the production of this manuscript.

\section{References}

1 Terry RD, Gonatas NK, Weiss M. Ultrastructural studies in Alzheimer's presenile dementia. Am. J. Pathol. 44, 269-297 (1964).

2 Englund E, Brun A, Alling C. White matter changes in dementia of Alzheimer's type. Biochemical and neuropathological correlates. Brain 111(Pt 6), 1425-1439 (1988).

3 Braak H, Braak E. Evolution of the neuropathology of Alzheimer's disease. Acta Neurol. Scand. Suppl. 165, 3-12 (1996).

4 Bartzokis G, Sultzer D, Lu PH, Nuechterlein KH, Mintz J, Cummings JL. Heterogeneous age-related breakdown of white matter structural integrity: implications for cortical "disconnection" in aging and Alzheimer's disease. Neurobiol. Aging 25(7), 843-851 (2004).

5 Ringman JM, O'Neill J, Geschwind D et al. Diffusion tensor imaging in preclinical and presymptomatic carriers of familial Alzheimer's disease mutations. Brain 130(Pt 7), 1767-1776 (2007).

6 Zhuang L, Sachdev PS, Trollor JN et al. Microstructural white matter changes in cognitively normal individuals at risk of amnestic MCI. Neurology 79(8), 748-754 (2012).
7 Mielke MM, Kozauer NA, Chan KC et al. Regionally-specific diffusion tensor imaging in mild cognitive impairment and Alzheimer's disease. Neuroimage 46(1), 47-55 (2009).

8 Teipel SJ, Stahl R, Dietrich O et al. Multivariate network analysis of fiber tract integrity in Alzheimer's disease. Neuroimage 34(3), 985-995 (2007).

9 Mielke MM, Leoutsakos JM, Corcoran CD et al. Effects of FDA approved medications for Alzheimer's disease on clinical progression. Alzheimers Dement. 8(3), 180-187 (2012).

10 Zhuang L, Wen W, Trollor JN et al. Abnormalities of the fornix in mild cognitive impairment are related to episodic memory loss. J. Alzheimers Dis. 29(3), 629-639 (2012).

11 Firbank MJ, Blamire AM, Teodorczuk A, Teper E, Mitra D, O’Brien JT. Diffusion tensor imaging in Alzheimer's disease and dementia with Lewy bodies. Psychiatry Res. 194(2), 176-183 (2011).

12 Kantarci K, Avula R, Senjem ML et al. Dementia with Lewy bodies and Alzheimer disease: neurodegenerative patterns characterized by DTI. Neurology 74(22), 1814-1821 (2010).
13 Laxton AW, Tang-Wai DF, McAndrews MP et al. A Phase I trial of deep brain stimulation of memory circuits in Alzheimer's disease. Ann. Neurol. 68(4), 521-534 (2010).

14 Smith GS, Laxton AW, Tang-Wai DF et al. Increased cerebral metabolism after 1 year of deep brain stimulation in Alzheimer disease. Arch. Neurol. 69(9), 1141-1148 (2012).

15 Teipel SJ, Wegrzyn M, Meindl T et al. Anatomical MRI and DTI in the diagnosis of Alzheimer's disease: a European multicenter study. J. Alzheimers Dis. doi:10.3233/JAD-2012-129904 (2012) (Epub ahead of print).

16 Oishi K, Mielke MM, Albert M, Lyketsos CG, Mori S. The fornix sign: a potential sign for Alzheimer's disease based on diffusion tensor imaging. J. Neuroimaging 22(4), 365-374 (2011).

17 Teipel SJ, Meindl T, Wagner M et al. Longitudinal changes in fiber tract integrity in healthy aging and mild cognitive impairment: a DTI follow-up study. J. Alzheimers Dis. 22(2), 507-522 (2010).

18 O'Dwyer L, Lamberton F, Bokde AL et al. Multiple indices of diffusion identifies white matter damage in mild cognitive impairment and Alzheimer's disease. PLoS ONE 6(6), e21745 (2011) 